\title{
ANALYSIS OF RASTER IMPRINTS PARAMETERS ON THE BASIS OF MODELS AND EXPERIMENTAL RESEARCH
}

\author{
Bohdan Durnyak ${ }^{1}$, Mikola Lutskiv ${ }^{1}$, Georgij Petriaszwili ${ }^{2}$ (D), Petro Shepita ${ }^{1}$ \\ ${ }^{1}$ Ukrainian Academy of Printing, Lviv, Ukraine \\ ${ }^{2}$ Warsaw University of Technology, Institute of Mechanics and Printing, Warsaw, Poland
}

\begin{abstract}
Structural scheme of models for determining the integral density of raster scale of an imprint, raster tone in percentage, optical density of scales on the basis of the measured optical density of the solid area and other characteristics have been worked out. The results of imitation design in the form of characteristics for different parameters have been provided. Optical density of raster scales obtained on the models has been found out to be quite similar to the results of experimental studies.
\end{abstract}

Key words: raster scale, imprints, models, optical density parameters, imitation design.

\section{PREFACE}

To represent graphical and text information by printing means, it is necessary to provide the high quality of an image that can lost during the preprint processes. It occurs during screening, production of printing plates and during printing itself due to different kinds of influences and distortions. Therefore, to provide quality of the printed products, it is necessary to control quality of implementation of separate technological operations and printing, to assess the optical density of raster image, in particular. An assessment is carried out mainly on experimental basis by means of densitometers on the basis of which graphic specifications of tone transfer are built, and analysis, synthesis, and correction of an image are maintained. Modern densitometers are devices that on the basis of measuring of intensity of the reflected or skipped light by certain algorithms determine optical density, relative area of the printed elements, their geometrical sizes, fineness, etc. (Pashulja, 2011; Predko, 2009). The producers of densitometers, for well-known reasons, do not present algorithms of calculation of separate parameters crucial for measurement accuracy that can be considerable. Therefore, it is a relevant task to compare simulation results on the basis of well-known expressions with experimental researches.

\section{BACKGRAUNDS}

In most cases, digital images used in printing are processed in the packages of computer graphics and visually perceived as qualitative ones (Blanter, 1999; Gonsales and Vuds, 2012; Vorobel, 2012). Converting of digital images into a raster form as well as printing plate making is carried out by CtP technology (Gonsales and Vuds, 2012; Vorobel, 2012). Quality of printing plate making can be experimentally assessed by means of a densitometer defining this or that parameter (Blanter, 1999; Pashulja, 2011; Predko, 2009). The existing methods of digital images processing use levels (gradations) of grey within limits [0, 255] and operate the arrays of numbers (Baranovskyj et al, 2013; Kuznetzov, 2002). Printing image, on the contrary, is much more complex: it has raster structure, is defined by an area of a raster (printed) element that has the set form of elements and fineness (Baranovskyj et al, 2013; Blanter, 1999; Lutskiv, 2012). Thus, it is impossible to apply various existing methods of digital images processing directly to raster images and raster printing (demodulation). To prepare qualitative image for printing, it is necessary to assess optical density of raster images, the area of raster elements and geometrical sizes. These days this estimation is carried out mainly experimentally by means of densitometers, gradation specifications of tone transfer are built on their basis as well as analysis, synthesis and correction of images are maintained, that makes it impossible to give objective estimation, optimization and anticipating of tone transfer on certain stages of preparation to printing (Baranovskyj et al, 2013; Lutskiv, 2012; Lutskiv and Buben, 2018). There is a formula of demodulation of raster transformation obtained by Murray and Davies and amendments of Yul - Nikols to this formula, however, the calculation of optical density is inaccurate and depends on ink, and paper properties as well as halftone frequency (Pashulja, 2011; Predko, 2009; Vorobel, 2012). In the work (Nazar et al, 2011) the authors represented a structural scheme of simulation model in Matlab package: Simulink, that makes it possible to calculate and build specifications of demodulation of linear raster scale for different fineness, define deviations of 
characteristics depending on halftone frequency and demodulation index, which is useful for practical applications. Gradation transforming of images tone in printing is mainly assessed by densitometry methods. For operative control and reproduction of raster tones multifunctional densitometers are widely used, that on the basis of measuring of intensity of the reflected or transmitted light by certain algorithms determine optical density of a solid tint, relative area of printing elements, tone, contrast, etc. (Pashulja, 2011; Predko, 2009). Optical density of a solid tint of printed (blackened) areas of an imprint is mostly assessed in number by a reflection optical density on the basis of reflectivity coefficient in a simplified way (Vorobel, 2012):

$$
D_{i}=\lg \frac{1}{R}
$$

Where $\mathrm{R}=\mathrm{F} / \mathrm{F}_{0}, \mathrm{~F}_{0}$ - intensity of the incident flow of light, $\mathrm{F}$ - intensity of the reflected light.

According to Weber-Fechner law the reaction of perception of the human visual system is proportional to logarithm of light stimulus (impulse). That is why the choice of optical density as measures of photographic darkening seems quite reasonable (Vorobel, 2012), as a change of optical density is proportional to lightness. A number of researches prove that non-linearity of human sight is characterized by a psychophysical function often described by logarithmic functions in semi-logarithmic coordinates. As optical density of a solid tone is determined in the reflected light, then measurement accuracy depends on uniformity of coverage of the scales fields, ink properties, whiteness of the solid tone (paper) (Pashulja, 2011). To increase the accuracy, it is necessary to take into account diffusion of a light stream, take $\mathrm{F} / \mathrm{n}$, where $\mathrm{n}$-is a coefficient, which characterizes the light diffusion.

The purpose of the article is to develop structural scheme of a model for determining the integral and optical densities of raster tone, to design special full format testing printing form, and to compare the results of simulation and experiment on the basis of simulation modelling, printing and experimental densitometry measurements.

\section{SUMULATION RESEARCHERS}

In printing reproduction, a solid tone is dispensed into raster elements in such a way that visually it corresponds to the value of the original tone. At densitometry control of raster imprints, a densitometer measures integral (raster, average) density that indicates the ability of light absorption by inked printing elements. On the basis of the raster scales of an imprint located on the fields, a densitometer determines an integral density. For this purpose it is necessary to the optical density (1) in a simplified version to add an expression of integral density in semi-logarithmic scale:

$$
D_{i}=\lg \frac{\phi_{0}}{\phi}, \text { if } 99 \leq \phi \leq 1
$$

where $\Phi_{0}=100 \%$ - incident flux of light, $\Phi \%$ - a light stream in percentage, reflected off the raster field. The majority of modern densitometers determine optical density and value of the raster tone. For instance, the value of the raster tone on a positive full tone (solid area) is $100 \%$, but the value of a raster tone without raster dots (paper) is $0 \%$. At alternate image the value of raster tone is $50 \%$ and it consists of half darkened area and half-light area.

The value of a positive of raster tone is determined by the formula (Predko, 2009):

$$
F_{r t}=\left[\Phi_{0}-\Phi\right] \%
$$

Integral density is subordinate to each value of raster tone:

$$
F_{r t}=\left(1-10^{D_{i i}}\right) 100 \%
$$

The integral density measured by the densitometer is the main output variable for which other indices of raster, printing production and printing are calculated. Simple densitometers determine the optical density of raster fields based on the measured optical density of the die by a simple expression (Pashulja, 2011):

$$
D_{M}=S \cdot D_{n}, \text { if } 0 \leq S \leq 1
$$


where Dn is the optical density of the solid area, $\mathrm{S}$ is the degree of coverage of the raster element with ink, which is physically close to the relative area of the raster element.

Densitometer does not directly measure the optical density of the solid area and the degree of coating of raster elements with ink, but determines them by one or other algorithms, so the accuracy depends on the chosen algorithm. Therefore, the convergence of the results of densitometry measurements can only be obtained for the same type of ink and its thickness, the same printed material under the same measurement conditions and using the same densitometer.

The calculation of the integral density of the imprint by expression (2) in the semi-logarithmic scale and the values of the raster tone according to the positive function (4), their comparison with the results of experimental studies is inconvenient, so on the basis of these expressions a structural diagram of the model was developed to determine the integral density of the raster imprint and the bitmap in Matlab package Simulink (Figure 1).

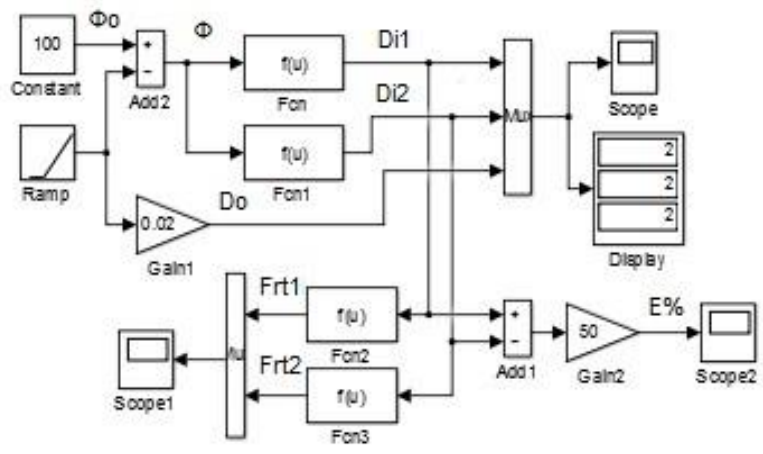

Figure 1: Structural diagram of integral density and raster tone model

The Constant and Ramp blocks form signal $F$ at the output of the summation block Add, which corresponds to the light flow reflected from the raster field and is fed to the output of the block of mathematical functions Fcn in a dialogue windows of which the program for calculating the integral bitmap density on the basis of expression (2) has been written. The second block of mathematical functions Fcn1 is similar to the previous one and additionally takes into account the individual effects on the integral density. The simulation results are visualized by Scope and Display blocks. To calculate the raster tone of the scales of expression (4), the block of mathematical functions Fcn2 and Fcn3 of multiplication and the block Constant were applied, to the inputs of which the calculated optical densities Di1 and Di2, located at the bottom of the scheme, are being fed.

Adjusted the Constant block to the stream falling $\Phi_{0}=100 \%$ and the Ramp block to the reflected stream $F$ according to expression (2). In block Fcn1 set the flux $F / n$, where $n=1,15$ - coefficient characterizing light scattering, which depends on the properties of paper and ink. The results of the simulation modelling of the integral density are presented in Figure 2.

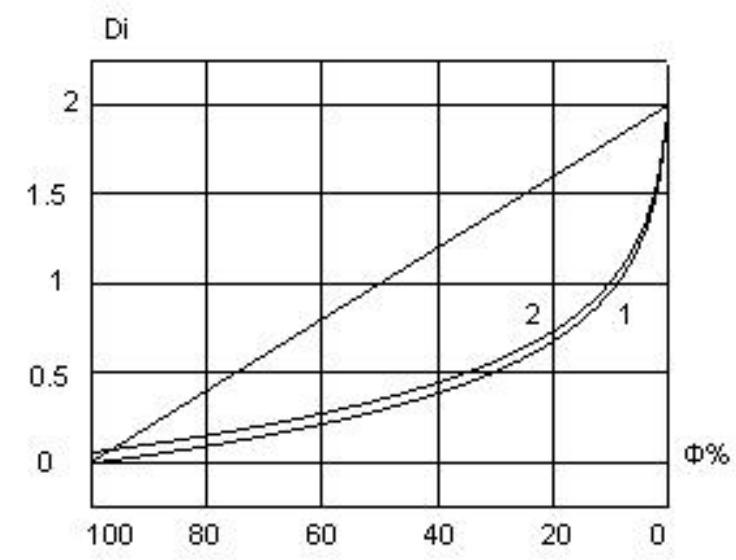

Figure 2: Characteristics of integral density

1 - calculated by expression (2), 2 - takes into account different influences 
For comparison, the linear characteristic $D_{0}$ is given in the figure 2. The peculiarities of features are their presentation on a semi-logarithmic scale. In addition, the beginning of the countdown of the reflected flux is reversed and begins with $\Phi=100 \%$, which is due to the method of measuring the integral density by the reflected light fluxes from the raster field of the raster scale of the print in which the image is formed in black. The designed characteristic is a rather non-linear curve, which is caused by logarithmic transformation (2). More precise results of the simulation are presented in Table 1 . The second characteristic takes into account different influences, for example, the paper's optical density or ink properties, which are higher than of the previous one. In order to evaluate the properties of the integral density objectively, it is suggested to determine its deviation from the previous one:

$E=\frac{D_{i} 1-D_{i}^{2}}{2} 100 \%$

which is determined by the Add1 block and is rendered by the Scope2 block. The results of the deviation of the integral density under the influence of the effects are presented in Figure 3.

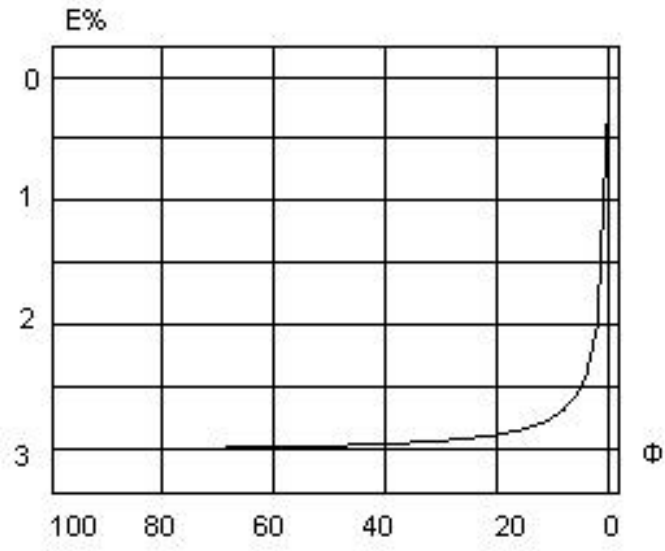

Figure 3: Deviation of integral density under the action of different influences

In light areas, the deviation of the integral density is $-3 \%$, and in the shadows $(\Phi<5 \%)$ it rapidly shifts to zero. As already noted, integral density is the main output variable by which other parameters are calculated, including the raster tone of the positive percentage (4), which is calculated in blocks of mathematical functions Fcn2 and Fcn3 based on the above calculated integral density, the results of which are visualized by Scope1. The simulation values of raster tone in percentage are calculated by the expression (4) and presented in Figure 4:

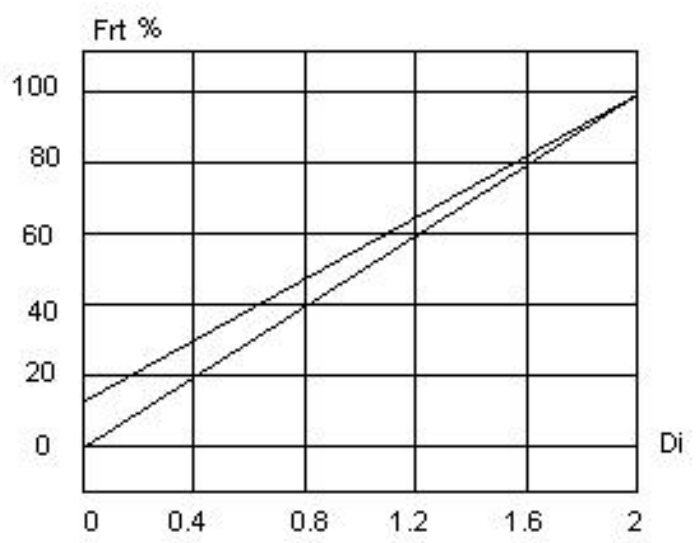

Figure 4: The characteristic of the raster tone of the positive is determined by the integral density 1-calculated by the expression (4), 2-with regards to different influences 
Characteristics of a raster tone under the action of various influences of the location are above the initial one. The maximum deviation is at a small integral density of $12.9 \%$, and at the end of the range gradually goes to zero. The percentage character of the raster tone is a straight line that is convenient for comparing the raster tone as a percentage on the films, printing plates and raster prints. Characteristics of a raster tone under the influence of various impacts of the location are above the initial one. The maximum deviation is at a small integral density of $12.9 \%$, and at the end of the range goes to zero. Results of simulation of optical linear density of raster scales based on the optical density of the plate by the simple expression (5) are given in Figure 5:

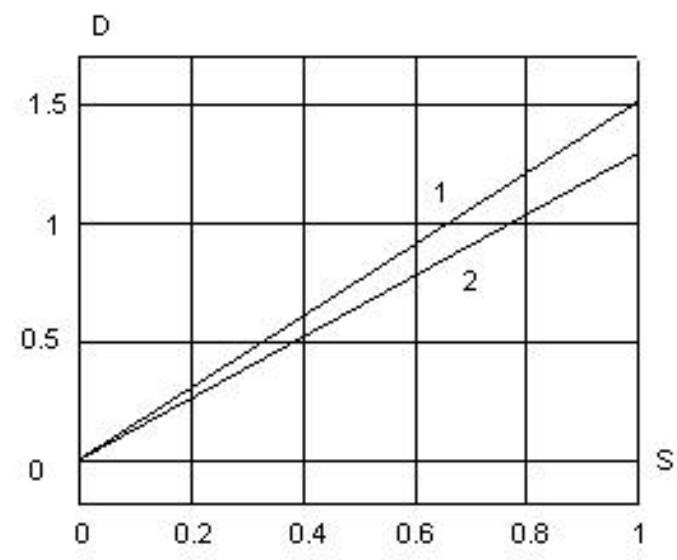

Figure 5: Optical density characteristics for different values the optical density of the solid area $1-$ for $D_{1}=1.52 ; 2-$ for $D_{2}=1.30$

At the beginning of the pitch range, the absolute tone difference is equal to zero, gradually increasing, and at the end of the range it is 0.22 . Therefore, increasing the thickness of the ink supply significantly affects the optical density of the print.

\section{EXPERIMENTAL RESEARCHERS}

As the manufacturers of densitometers for known reasons do not provide algorithms and expressions for calculations, certain indicators may be different Therefore, the task is to equate and compare the results of simulation modelling obtained from the expressions (2) - (5) of experimental studies. To achieve this goal, a special testing multipurpose raster printing plate has been developed. Typically, traditional tests are intended for the operational control and adjustment of tone transmission at a given circulation, so they are small in size and are located in places of further bending of the sheet when it is folded or at its edge. Therefore, the thickness of the ink on the text boxes may be much larger than on the surface of the print, which is the disadvantage of such small-format texts, which can lead to distorted results of experimental studies. Therefore, a special full-format test form has been developed for research, which ensures adequate experimental studies. The test typeface contains a sequence of stripes whose height is equal to the height of the shape. The width of the strips corresponds to the step of placing the ink supply onto the strip. The strips contain continuous raster fields of 5, 10, 20, 40, 60, 80, 90 and 100\% tonal graduation and small-format $100 \%$ solid areas for measurement. The test form is made using CtP technology, from the computer to the plate, providing greater accuracy in the production of the plate. The results of the experimental studies were performed on offset printing machine ADAST Dominant 525 format B3 521x415mm. Printing was done with Michel Huber Rapida Black ink on $120 \mathrm{~g} / \mathrm{m}^{2}$ coated paper. The zonal supply of the inking unit to the test plate at the $40^{\circ}$ rotation angle of the ink fountain roller has been adjusted. Press proofing has been completed, then a print check of test prints has been performed. The measurement of the optical density of the raster fields and solids has been performed using a X-Rite 504 digital spectral densitometer. The averaged results of the optical density measurements on the bands are presented in the table. After that, the second stage of the experimental studies has been carried out at a maximum angle of rotation of ink fountain roller by $170^{\circ}$. From the table we can conclude that the optical density on the test strips depends significantly on the angle of rotation of the ink fountain roll and stretches the dynamic range of the transmission from 0.06 to $1.30 \mathrm{D}$ and from 0.08 to $1.51 \mathrm{D}$. 
The optical density of raster fields has been calculated on the basis of experimental studies of the optical densities of the solid area by expression (5), provided that the degree of ink coating of the raster element is equal to the degree of coverage of the raster strips, and the calculation results are presented in the Table 1:

Table 1: Measurement and simulation results

\begin{tabular}{|c|c|c|c|c|c|c|c|}
\hline $\begin{array}{l}\text { Tonal } \\
\text { graduation }\end{array}$ & \multicolumn{2}{|l|}{$\begin{array}{l}\text { Measured optical } \\
\text { density of scales }\end{array}$} & \multicolumn{2}{l|}{$\begin{array}{l}\text { Absolute } \\
\text { difference }\end{array}$} & \multicolumn{2}{l|}{$\begin{array}{l}\text { Calculated optical } \\
\text { density of scales }\end{array}$} & \multicolumn{2}{l|}{$\begin{array}{l}\text { Absolute difference of } \\
\text { densities }\end{array}$} \\
\hline S\% & D1 & D2 & $\Delta$ D & $D_{\text {H1 }}$ & $D_{\text {H2 }}$ & $\Delta D 1$ & $\Delta D 2$ \\
\hline 5 & 0,06 & 0,08 & 0,02 & 0,065 & 0,075 & $-0,005$ & 0,015 \\
10 & 0,15 & 0,18 & 0,03 & 0,13 & 0,151 & 0,02 & 0,03 \\
20 & 0,30 & 0,36 & 0,06 & 0,26 & 0,302 & 0,04 & 0,06 \\
40 & 0,51 & 0,64 & 0,13 & 0,52 & 0,604 & $-0,01$ & 0,04 \\
60 & 0,83 & 0,95 & 0,12 & 0,78 & 0,906 & 0,05 & 0,044 \\
80 & 1,14 & 1,35 & 0,21 & 1,04 & 1,208 & 0,10 & 0,14 \\
90 & 1,21 & 1,46 & 0,25 & 1,17 & 1,359 & 0,04 & 0,01 \\
100 & 1,30 & 1,51 & 0,21 & 1,30 & 1,520 & 0 & 0 \\
\hline
\end{tabular}

According to the tabular data of the results of measuring the optical densities of the raster scales, we conclude that increasing the thickness of the ink transfer by increasing the angle of rotation of the ink fountain roller significantly affects the optical density of the raster scales. In the middle tones the absolute difference in optical densities $D 2-D 1=\Delta D$ is in the range 0.1-0.2, and in the dark tones $\Delta D=0.2-$ 0.25. Therefore, the thickness of the ink is a major factor influencing the optical density of images. Therefore, the inking unit of the offset printing machine could be adjusted using a multi-channel automatic zonal feed system for each circulation in particular. The optical density of the raster scales, calculated by the simple expression (5), is quite similar to the results of experimental studies and practically independent of the studies and of the thickness of the ink layer. The absolute difference of the optical densities of the scales is in the range $\Delta D=D I-D M$, and in the range -0.01 to 0.14 . The National Standards of Ukraine - DSTU:ISO 12647-2:2005 recommend a list of indicators for offset printing at an optical density of D from 1.4 to 1.6 in which the optical density of raster scales for chalked paper is \pm 0.15 . Therefore, the calculation of the optical density of the scales on the basis of the simple model (5) provides the required accuracy.

\section{CONCLUSIONS}

Densitometers do not directly measure optical density, the degree of coverage of raster scales, and other parameters, but determine them by one or another, in most unknown algorithms, on which the accuracy of measurement depends. The models of integral (average) density are presented on a semi-logarithmic scale, and the values of the raster tone of the positive, used in densitometers operating in reflected light, and the relationships between them, have been justified. The structural scheme of the model is developed to determine the integral density of raster scales of the imprint, raster tone in percentage, optical density of scales on the basis of the measured optical density of the solid area, which calculate and construct their characteristics. The simulation results are presented in the form of characteristics for different parameters.

For the experimental research of the simulation results, a special proof plate with wide strips - with raster scales - has been developed to ensure adequate experimental research. The results of the measurements of the optical density of the scales and the results of the measurements have been summarized in the table, on the basis of which it has been concluded that an increase in the thickness of the ink supply significantly affects the optical density of the scales. On the middle tones, the absolute difference in optical densities is in the range 0.1-0.2, and on the dark tones it is 0.2-0.25. The optical density of the raster scales obtained on the models is quite similar to the results of experimental studies and practically does not depend on the thickness of the ink layer. The absolute difference of optical densities is in the range from 0.01 to 0.14 , which corresponds to the tolerance of the optical densities of raster scales for chalked paper \pm 0.15 , which ensures the accuracy of modelling. 


\section{REFERENCES}

[1] Baranovskyj V., Lutskiv M., Fil L., Chernozubova G.: "Construction and analysis of screening characteristics", Journal Naukovi zapysky UAP 4, 131-138, 2013.

[2] Blanter D.: "Scanning and screening images", (ECOM, Moscow, 1999.)

[3] Gonsales R., Vuds R.: "Digital image processing", (Tekhnosfera, Moscow, 2012.)

[4] Kuznetzov J.: "Technology for image information processing", (Petersburg Institute of Printing, St. Petersburg, 2002.)

[5] Lutskiv M.: "Digital printing technology", (Ukrainian Academy of Printing, Lviv, 2012.)

[6] Lutskiv M., Buben V.: "Construction of the characteristics of demodulation linear tint chart", Journal Computer printing technology 2 (40), 2018, 109-115.

[7] Nazar I., Lazarenko E., Jakutsevych S.: "Offset printing options: management and influence factors", (UAP, Lviv, 2011.)

[8] Pashulja P.: "Standardization, metrology, compliance, printing quality", (UAP, Lviv, 2011.)

[9] Predko L.: "Prepress Process Design: tutorial", (UAP, Lviv, 2009.)

[10] Vorobel R.: "Logarithmic image processing", (Naukova dumka, Kyiv, 2012.)

(C) 2020 Authors. Published by the University of Novi Sad, Faculty of Technical Sciences, Department of Graphic Engineering and Design. This article is an open access article distributed under the terms and conditions of the Creative Commons Attribution license 3.0 Serbia (http://creativecommons.org/licenses/by/3.0/rs/). 